\title{
Percepção do estudante sobre a influência do ambiente/clima educacional no processo ensino- aprendizagem: uma revisão integrativa
}

Student's perception of the influence of the educational environment / climate in the teaching-learning process: an integrative review

Percepción de los estudiantes de la influencia del medio ambiente / clima educativo en el proceso de enseñanza y aprendizaje: una revisión integrativa

Milena da Costa ${ }^{1}$, Ivete Palmira Sanson Zagonel ${ }^{2}$

\footnotetext{
${ }^{1}$ Mestre no Ensino nas Ciências da Saúde pela Faculdades Pequeno Príncipe - FPP. Curitiba -

Paraná.

${ }^{2}$ Doutora em Enfermagem pela Universidade Federal de Santa Catarina. Docente do Programa de Mestrado em Ensino nas Ciências da Saúde da Faculdades Pequeno Príncipe - FPP. Curitiba Paraná.
}

\section{RESUMO}

Trata-se de revisão integrativa que objetivou descrever o conceito de ambiente/clima educacional e identificar a influência do ambiente/clima educacional no processo ensino-aprendizagem sob a percepção do estudante. Ambiente educacional (AE) é todo e qualquer contexto em que se dá o ensino e o aprendizado. Foram selecionados doze artigos relevantes ao tema, nas bases de dados BVS e PubMed, com descritores, educação superior, competência clínica, procedimentos clínicos e aprendizagem. Emergiram três categorias: ambiente educacional e ensino clínico na

Autor de Correspondência:

*Milena da Costa . E-mail: mi.enfermagem.fpp@gmail.com 
saúde; percepção do estudante sobre o ambiente educacional e fatores do ambiente educacional que influenciam no processo ensino-aprendizagem. $\mathrm{O} A E$ pode afetar as habilidades e $\mathrm{O}$ desempenho acadêmico e deve propiciar confiança nas atividades diárias. Conclui-se que o AE tem influência no processo ensino-aprendizagem, fornece informações importantes para os educadores, estimulam a autoconfiança e aspectos afetivos.

Palavras-chave: Educação Superior. Competência Clínica. Procedimentos Clínicos. Aprendizagem.

\section{ABSTRACT}

This is an integrative review, which aimed to describe the concept of educational environment / climate and to identify the influence of the educational environment / climate on the teachinglearning process according to the student's perception. Educational environment (AE) is any and all contexts in which teaching and learning take place. Twelve articles relevant to the topic were selected in the VHL and PubMed databases, with descriptors educational environment, health education, learning in clinical teaching, education environment AND health education, learning in clinical teaching. Three categories emerged: educational environment and clinical teaching on health; student's perception of the educational environment, and factors from the educational environment that influence the teaching-learning process. AE can affect academic skills and performance and should provide confidence in daily activities. It is concluded that $\mathrm{AE}$ has some influence on the teaching-learning process, provides important information for educators, stimulates self-confidence, and affective aspects.

Keywords: Education, Higher. College Education. Clinical Competence. Critical Pathways. Clinical Procedures. Learning

\section{RESUMEN}

Esta es una revisión integradora, cuyo objetivo fue describir el concepto de ambiente / clima educativo e identificar la influencia del ambiente / clima educativo en el proceso de enseñanzaaprendizaje bajo la percepción del estudiante. El entorno educativo (AE) es cualquier contexto en el que se lleva a cabo la enseñanza y el aprendizaje. Doce (12) artículos relevantes para el tema fueron seleccionados en las bases de datos de BVS y PubMed, con descriptores, educación universitaria, competencia clínica, procedimientos clínicos y aprendizaje. Surgieron tres (3) categorías: ambiente educativo y enseñanza clínica en salud; percepción del alumno del entorno educativo y los factores del entorno educativo que influyen en el proceso de enseñanzaaprendizaje. AE puede afectar las habilidades académicas y el rendimiento y debe proporcionar confianza en las actividades diarias. Se concluye que la EA influye en el proceso de enseñanzaaprendizaje, proporciona información importante para los docentes, estimula la autoconfianza y los aspectos afectivos.

Palabras clave: Educación Superior. Universitaria. Competencia Clínica. Vías Clínicas. Procedimientos Clínicos. Aprendizaje. 


\section{INTRODUÇÃO}

O conceito de ambiente educacional (AE) é todo e qualquer contexto em que se dá o ensino e o aprendizado. Sinônimos que estão sendo utilizados para ambiente educacional são "atmosfera educacional" e "clima educacional"1. O ambiente educacional deve proporcionar aos estudantes oportunidade para aprender com uma relação entre o estudante, docentes e membros da equipe de saúde, que estimulem a autoconfiança, favorecendo o processo de aprendizagem ${ }^{2}$.

O ensino em saúde não ocorre somente em sala de aula e sim, em ambientes, cenários da prática profissional de cada área, favorecendo a interação dos docentes, estudantes, profissionais de serviços e usuários. Essas formas de ensino são necessárias, e uma complementa a outra de forma a oferecer melhores oportunidades de aprendizagem ao estudante ${ }^{3}$.

Diversos fatores influenciam no processo ensinoaprendizagem, como instalações, estrutura física e material, o relacionamento interpessoal, a metodologia de ensino, as dimensões ética e social, serviços acadêmicos, programas de assistência social, política de avaliação institucional, qualificação docente, qualificação técnico-administrativa, relação docente/estudante, entre outros. Essas influências integram elementos constitutivos, favorecendo a formação de um profissional crítico e reflexivo, reconhecendo o estudante como sujeito ativo. O ambiente educacional deve estimular o estudante no convívio social e no relacionamento interpessoal e, apoiá-lo em atividades intra e extra universitárias ${ }^{3,4}$. Estimular através da autonomia e responsabilidade, proporcionar oportunidades para executar diferentes tarefas, apoio e feedback do desempenho do estudante por preceptores ${ }^{2}$.

As expressões ambiente educacional, clima educacional e ambiente de aprendizagem são consideradas sinônimos. O clima educacional na percepção dos estudantes tem impacto na qualidade de aprendizagem ${ }^{1}$.
A percepção do estudante sobre o ambiente educacional está relacionada a questões subjetivas, expectativas e conceitos prévios de satisfação e sucesso, considerada um fator determinante no processo ensino-aprendizagem. $\mathrm{O}$ ambiente educacional pode influenciar diretamente $o$ desempenho acadêmico do estudante em seu período de formação. Avaliar o ambiente educacional pela percepção dos estudantes pode fornecer informações importantes para a melhoria do processo ${ }^{5}$.

No ambiente escolar, a sala de aula é o local onde os estudantes permanecem maior tempo em sua rotina diária. Este proporciona a criação de vínculos uns com os outros, além do desenvolvimento biopsicossocial, crescimento não só cognitivo, mas pessoal, literal e emocional, desenvolvendo a relação dos estudantes com a aprendizagem. Porém, a forma como o estudante percebe e se relaciona neste espaço pode repercutir em outras áreas de sua vida, interferindo também em sua formação ${ }^{6}$.

Fatores que podem dificultar o processo de aprendizagem incluem a falta de confiança nos estudantes, demonstradas pelo docente, escassez de oportunidades para realizar procedimentos práticos e sentimentos de baixa autoconfiança entre os estudantes ${ }^{2}$.

Estudos revelam que investir em melhorias estruturais no ambiente educacional pode aumentar a eficiência e a produtividade acadêmica dos estudantes. A readequação do ambiente pode ser uma estratégia eficaz para atingir os objetivos organizacionais, pois, contempla tanto as necessidades da instituição de ensino, como as expectativas e ideias pessoais do estudante, eliminando os efeitos negativos de insatisfação com o ambiente formativo e o curso ${ }^{4}$.

As percepções dos estudantes sobre a qualidade do ambiente educacional, revelam informações importantes para os educadores relacionadas ao processo ensino-aprendizagem. Com o intuito 
de nortear esta pesquisa tem-se como objetivos descrever o conceito de ambiente/clima educacional e identificar a influência do ambiente/clima educacional no processo ensino-aprendizagem sob a percepção do estudante ${ }^{2}$.

\section{MÉTODO}

Trata-se de uma revisão integrativa seguindo o referencial proposto por Mendes, Silveira e Galvão (2008; 2019), através da análise ampla de pesquisas relevantes, que dão suporte para a tomada de decisão e a melhoria da prática clínica. Tem o potencial de construir conhecimento em enfermagem, produzindo um saber fundamentado e uniforme para os enfermeiros realizarem uma prática clínica de qualidade. Além disso, pode reduzir alguns obstáculos da utilização do conhecimento científico, tornando os resultados de pesquisas mais acessíveis, uma vez que em um único estudo o leitor tem acesso a diversas pesquisas realizadas. Ou seja, o método permite agilidade na divulgação do conhecimento ${ }^{7,8}$.
A revisão integrativa, segundo referencial metodológico apresentado, recomenda a utilização de 6 (seis) etapas para o seu desenvolvimento. $\mathrm{Na}$ primeira etapa foi delimitado o tema de estudo e elaboração da pergunta da revisão, sendo ela: "qual a influência do ambiente/clima educacional no processo ensino-aprendizagem, pela percepção dos estudantes da área de saúde?”. Na segunda, foi realizada a busca e seleção dos estudos primários na Biblioteca Virtual da Saúde (BVS) e PubMed, utilizando os seguintes descritores: Ambiente educacional e Ensino na saúde, Aprendizagem no ensino clínico, Educational environment AND health education, Learning in clinical teaching. Foram utilizados os seguintes critérios de inclusão: estudos disponíveis na íntegra, nos idiomas português e inglês, publicados nos últimos 5 anos, ou seja, dos anos de 2014 a 2018. Os critérios de exclusão foram: teses e dissertações, resumos, artigos não disponíveis, outros idiomas, fora do período estabelecido, artigos redundantes e não relevantes ao tema.

Após aplicar os critérios de inclusão, em quatro buscas realizadas, obteve-se, conforme Figura 1.

Figura 1 - Busca de artigos nas bases de dados BVS e PubMed

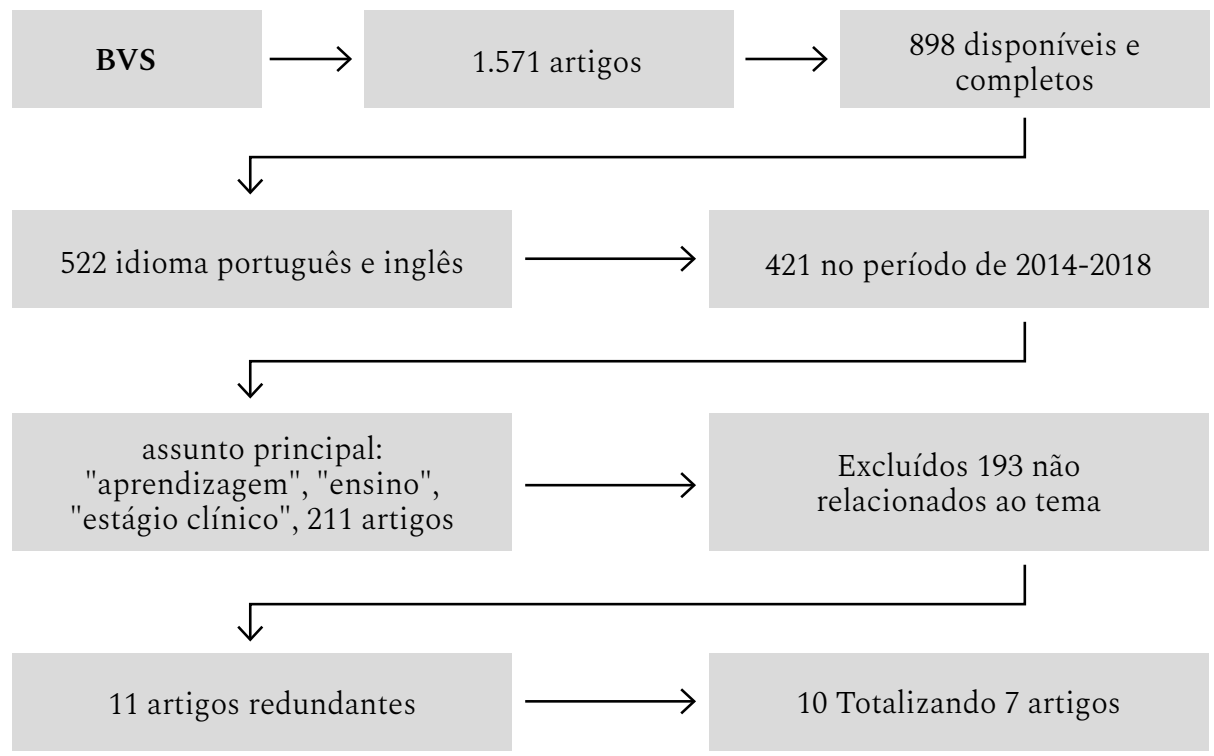




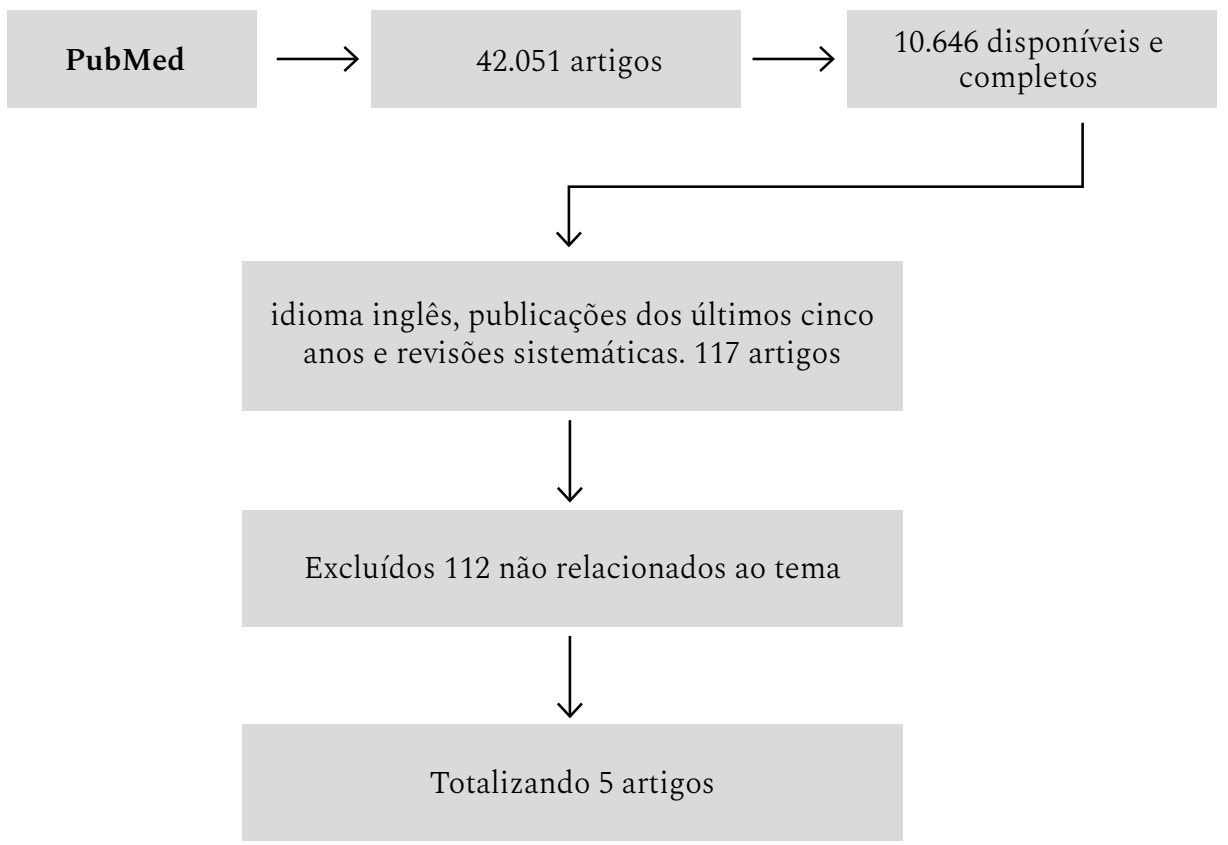

Fonte: a pesquisa.

$\mathrm{Na}$ busca de artigos pela plataforma da Biblioteca Virtual da Saúde (BVS), foram recuperados 1.571 artigos; destes, 898 estavam disponíveis na íntegra e completos; após aplicar critérios como idiomas português e inglês, 522 artigos; dentro do ano estipulado de publicação, 421 artigos; após aplicar demais critérios como assunto principal com os descritores "educação superior", "competência clínica", "procedimentos clínicos” e "aprendizagem” foram recuperados 211 artigos; após a leitura dos títulos e resumos, foram excluídos 193 não relacionados ao tema e 11 redundantes, totalizando 7 (sete) artigos que constituíram uma parte da amostra desta revisão.

$\mathrm{Na}$ busca de artigos pela plataforma PubMed, foram recuperados o total de 42.051 artigos; destes 10.646 estavam disponíveis na íntegra e completos, após aplicar critérios como idioma inglês, publicações dos últimos cinco anos e revisões sistemáticas, foram encontrados 117 artigos; após a leitura dos títulos e resumos, foram excluídos 112 artigos não relacionados ao tema, totalizando 5 (cinco) artigos que constituíram outra parte da amostra.

As buscas pela BVS e PubMed totalizaram 12 (doze) artigos que constituíram a amostra final desta revisão, os quais foram analisados na íntegra.

\section{RESULTADOS}

A Tabela 1 apresenta os estudos selecionados, descrevendo os autores, título, o ano e o periódico de publicação de cada artigo. 
Tabela 1 - Distribuição segundo título, autores, ano de publicação, periódico dos artigos encontrados.

\begin{tabular}{|c|c|c|c|c|}
\hline № & AUTORES & TÍTULO & $\begin{array}{c}\text { ANO DE } \\
\text { PUBLICAÇÃO }\end{array}$ & PERIÓDICOS \\
\hline 1 & Troncon, L.E.A. & Ambiente Educacional. & 2014 & $\begin{array}{l}\text { Medicina (Ribeirão } \\
\text { Preto) }\end{array}$ \\
\hline 2 & $\begin{array}{l}\text { Merighi, M.A.B. } \\
\text { et al. }\end{array}$ & $\begin{array}{l}\text { Ensinar e aprender no campo clínico: perspectiva } \\
\text { de docentes, enfermeiras e estudantes de } \\
\text { enfermagem. }\end{array}$ & 2014 & $\begin{array}{l}\text { Revista Brasileira } \\
\text { de Enfermagem }\end{array}$ \\
\hline 3 & $\begin{array}{l}\text { Colbert-Getz, J.M. } \\
\text { et al. }\end{array}$ & $\begin{array}{l}\text { Assessing Medical Students' and Residents' } \\
\text { Perceptions of the Learning Environment: } \\
\text { Exploring Validity Evidence for the } \\
\text { Interpretation of Scores From Existing Tools. }\end{array}$ & 2014 & $\begin{array}{l}\text { Academic } \\
\text { Medicine }\end{array}$ \\
\hline 4 & Mourão, L.F.et al. & $\begin{array}{l}\text { Perspectiva dos discentes de enfermagem sobre o } \\
\text { estágio curricular. }\end{array}$ & 2015 & $\begin{array}{l}\text { Revista de } \\
\text { Enfermagem UFPI }\end{array}$ \\
\hline 5 & $\begin{array}{l}\text { Baecher-Lind, L.E.; } \\
\text { Chang, K.; Blanco, } \\
\text { M.A. }\end{array}$ & $\begin{array}{l}\text { The learning environment in the obstetrics } \\
\text { and gynecology clerkship: an exploratory study } \\
\text { of students' perceptions before and after the } \\
\text { clerkship. }\end{array}$ & 2015 & $\begin{array}{l}\text { Medical Education } \\
\text { Online }\end{array}$ \\
\hline 6 & $\begin{array}{l}\text { Orsini, C.; Evans, } \\
\text { P.; Jerez, O. }\end{array}$ & $\begin{array}{l}\text { How to encourage intrinsic motivation in the } \\
\text { clinical teaching environment?: a systematic } \\
\text { review from the self-determination theory. }\end{array}$ & 2015 & $\begin{array}{l}\text { Journal of } \\
\text { Educational } \\
\text { Evaluation for } \\
\text { Health Professions }\end{array}$ \\
\hline 7 & Barrett, A. et al. & $\begin{array}{l}\text { A BEME (Best Evidence in Medical Education) } \\
\text { systematic review of the use of workplace-based } \\
\text { assessment in identifying and remediating poor } \\
\text { performance among postgraduate medical } \\
\text { trainees. }\end{array}$ & 2015 & Systematic Reviews \\
\hline 8 & $\begin{array}{l}\text { Yeh, T.K.; Huang, } \\
\text { H.M.; Chan, W.P.; } \\
\text { Chang, C.Y. }\end{array}$ & $\begin{array}{l}\text { Effects of congruence between preferred and } \\
\text { perceived learning environments in nursing } \\
\text { education in Taiwan: a cross-sectional study. }\end{array}$ & 2016 & BMJ Open \\
\hline 9 & $\begin{array}{l}\text { Mogre, V. Amalba, } \\
\text { A. }\end{array}$ & $\begin{array}{l}\text { Psychometric Properties of the Dundee Ready } \\
\text { Educational Environment Measure in a Sample of } \\
\text { Ghanaian Medical Students. }\end{array}$ & 2016 & $\begin{array}{l}\text { Education for } \\
\text { Health }\end{array}$ \\
\hline 10 & Silva, E.F.L. et al. & $\begin{array}{l}\text { Competências do docente do ensino clínico no } \\
\text { curso de graduação em enfermagem: um estudo } \\
\text { de caso. }\end{array}$ & 2017 & $\begin{array}{l}\text { Revista de } \\
\text { Enfermagem UFPE } \\
\text { on line }\end{array}$ \\
\hline 11 & $\begin{array}{l}\text { Hägg-Martinell, } \\
\text { A.; Hult, H.; } \\
\text { Henriksson, P.; } \\
\text { Kiessling, A. }\end{array}$ & $\begin{array}{l}\text { Medical students' opportunities to participate } \\
\text { and learn from activities at an internal medicine } \\
\text { ward: an ethnographic study. }\end{array}$ & 2017 & BMJ Open \\
\hline 12 & Visser, C.L.F. et al. & $\begin{array}{l}\text { Perceptions of residents, medical and nursing } \\
\text { students about Interprofessional education: } \\
\text { a systematic review of the quantitative and } \\
\text { qualitative literature. }\end{array}$ & 2017 & $\begin{array}{l}\text { BMC Medical } \\
\text { Education }\end{array}$ \\
\hline
\end{tabular}


Após a leitura e análise dos artigos selecionados foi possível obter resultados quantitativos que são apresentados de forma descritiva. Com relação ao ano de publicação dos artigos selecionados para o estudo, tem-se que os anos com maior número de publicações foram 2015, com quatro artigos (33,3\%); seguido pelos anos de 2014 e 2017, com três (25\%) artigos publicados cada um; em 2016, foram publicados dois $(16,7 \%)$ artigos relacionados ao tema.

Quanto aos periódicos em que foram publicados os artigos selecionados na pesquisa, tem-se que na revista $B M J$ Open foram publicados dois $(16,7 \%)$ artigos; e, nos demais periódicos foram publicados um (8,3\%) artigo em cada, sendo eles: Medicina (Ribeirão Preto), Education for Health, Revista de Enfermagem UFPE on line, Revista Brasileira de Enfermagem, Revista de Enfermagem UFPI, Medical Education Online, Systematic Reviews, Academic Medicine, Journal of Educational Evaluation for Health Professions, BMC Medical Education.

Com relação ao tipo de estudo, quatro utilizaram a revisão sistemática (33,3\%); e os demais estudos utilizaram abordagem quantitativa; estudo transversal; qualitativo, descritivo e exploratório; qualitativo com abordagem da fenomenologia; exploratório descritivo; qualitativo e etnográfico, ambos com um $(8,3 \%)$ artigo publicado em cada tipo de estudo.

Os participantes dos estudos das pesquisas foram estudantes de enfermagem, estudantes e residentes de medicina, docentes e enfermeiras das instituições. Os locais realizados foram ambientes hospitalares e instituições de ensino superior.

\section{DISCUSSÃO}

Após a leitura e análise dos artigos selecionados para este estudo, foi possível organizá-los em três categorias: ambiente educacional e ensino clínico na saúde; percepção do estudante sobre o ambiente educacional e fatores do ambiente educacional que influenciam no processo ensino-aprendizagem.

\section{Categoria I - Ambiente educacional e ensino clínico na saúde}

O ambiente educacional pode ser definido como um conjunto de elementos, de ordem material ou afetiva, que está em volta do educando em seu processo ensino-aprendizagem, sendo possível vivenciar os processos de ensino e aprendizado, exercendo influência definida sobre a qualidade do ensino e a eficácia do aprendizado. O educando é um elemento que participa do ambiente, atribuindo como responsabilidades a manutenção e o aperfeiçoamento deste ambiente ${ }^{1}$.

Quando se fala em ambiente educacional, inclui-se a relação do estudante-docente, as estratégias de ensino e aprendizagem, as necessidades psicossociais e emocionais dos estudantes, as estruturas físicas e instalações fornecidas pela instituição, propiciando um ambiente de aprendizagem produtivo ao estudante. $\mathrm{O}$ estudo ainda relata que o ambiente pode afetar as competências dos estudantes, o seu desempenho acadêmico e o seu sucesso futuro. Um estudo ainda ressalta que parte importante dos componentes do ambiente educacional é a que se relaciona ao ambiente físico em que se dá o aprendizado ou às condições materiais que cercam o ensino e o aprendizado9. Os estudantes devem estar confortavelmente acomodados em espaço físico adequado que permita desenvolver as atividades propostas $^{9}$.

Devido a isso, o ambiente educacional tem sido um dos alvos de avaliação de programas de educação médica pela Organização Mundial e Federação de Educação Médica. Com isso, várias ferramentas e instrumentos foram desenvolvidos para avaliar estes ambientes pela percepção do estudante ${ }^{9}$.

Além de um "clima educacional" adequado, os docentes/supervisores devem ser competentes e 
entusiasmados, de modo a motivar o estudante, oferecendo-lhe oportunidades de interagir com os pacientes e permitir que ele assuma a responsabilidade. Proporcionando ao estudante formar sua própria identidade profissional. Com isso, os objetivos de aprendizagem, a parceria com os supervisores e a continuidade são aspectos fundamentais para um ambiente de aprendizagem estimulante. Como descrito em outros estudos, o bom relacionamento do supervisor com o estudante e uma orientação eficaz são imprescindíveis no processo ensino-aprendizagem ${ }^{10}$.

O ensino clínico tem sido sugerido como uma modalidade de ensino que influencia a motivação intrínseca e o desempenho dos estudantes. Os estudantes precisam observar as tendências naturais para aprender e conhecer o ambiente que os cerca, ser responsáveis por suas próprias decisões e ter a possibilidade de apreender determinadas situações para enfrentar o seu dia a dia, sendo oportunizado no ensino clínico.

Estudos ainda evidenciam que se os docentes apoiarem os estudantes, desenvolvendo a autonomia, competência e o relacionamento, eles conseguirão assumir a responsabilidade por seu aprendizado e agirão de maneira mais autônoma com os pacientes no ambiente educacional em que está inserido ${ }^{11}$.

O ensino clínico é um componente vital para que o estudante possa colocar em prática os conhecimentos introduzidos em sala de aula, proporcionando o desenvolvimento das habilidades e competências clínicas que devem compor o perfil do profissional de saúde, tornando-se um profissional crítico e reflexivo, com conhecimentos científicos necessários ao desenvolvimento do cuidado ${ }^{12}$.

\section{Categoria II - Percepção do estudante sobre o ambiente educacional}

A percepção dos estudantes sobre o ambiente de aprendizagem tem sido de suma importância, tem como objetivo avaliar este ambiente/clima educacional e a forma que os estudantes percebem e interpretam um ambiente de aprendizagem. Fatores estes que podem influenciar o seu conhecimento prévio, experiências e habilidades ${ }^{13}$.

No estudo realizado em uma instituição pública de ensino, os estudantes relataram que a infraestrutura física da instituição em que realizaram o estágio curricular atendia parcialmente as necessidades para as atividades propostas. Em outra pesquisa, os estudantes relataram que estar ativamente engajados no processo de aprendizado, receber supervisão clínica adequada e valorizar os membros da equipe para ajudar a dar suporte ao atendimento ao paciente foram expectativas dos estudantes pré-matriculados em um estágio supervisionado de obstetrícia e ginecologia. Porém, eles também relataram que tiveram que trabalhar "duas vezes mais" para ganhar o respeito e participação da equipe na sala obstétrica $^{14,15}$.

Estudo realizado em uma enfermaria com estudantes de medicina, no momento da visita, o supervisor realizava perguntas aos estudantes. Nesse momento, eles expressavam um sentimento de pressão para demonstrar seu conhecimento, mostrar que estavam curiosos e que tinham interesse em aprender mais. Durante essa rodada de perguntas, alguns estudantes se sentiam desconfortáveis e ansiosos. Porém, ao se passar um longo período durante o programa médico, eles disseram que se sentiam mais à vontade com seu próprio aprendizado e sobre como atuavam em várias situações clínicas. Eles ainda relataram no estudo que esse progresso era óbvio entre o quinto e o sexto semestre. Achavam que seu progresso era facilitado pelas oportunidades de se envolver no atendimento direto ao paciente ${ }^{16}$.

$\mathrm{Na}$ pesquisa realizada em uma universidade central de Taiwan com estudantes de enfermagem, estes relataram que durante algumas atividades propostas pelo docente, "eram obrigados a discutir, pensar e compartilhar ideias em um período de 
tempo limitado", "não podiam pensar e discutir cada item com detalhes" e "sentiam uma pressão impressionante". Com isso, sentiam que poderiam não ser capazes de pensar em profundidade suficiente para satisfazer as expectativas do docente ${ }^{13}$ Ainda falando sobre a percepção dos estudantes sobre o ambiente educacional que estão inseridos, em um outro estudo, os estudantes de medicina que completaram o estágio supervisionado, descreveram ser um ambiente de aprendizagem passivo em que se sentiram isolados, desvalorizados e desnecessariamente excluídos das atividades clínicas ${ }^{15}$.

Alguns estudos relatam que os estudantes de enfermagem no ensino clínico sentiam que os docentes os tratavam como adolescentes, em vez de adultos, afetando sua motivação. Os docentes devem apoiar um ambiente no qual os estudantes se sintam emocionalmente apoiados, confortáveis e livres para expressar suas opiniões, motivando-os e despertando o interesse pelas disciplinas e atividades propostas. Alegaram também que, ao dizer algo crítico, os docentes agiam se defendendo ao invés de ouvir e discutir a situação. O docente precisa ser empático ao ouvir as opiniões dos estudantes. Estudos ressaltaram a importância de se respeitar e ter uma atitude positiva em relação aos estudantes, proporcionando um ambiente não ameaçador, saudável e confortável em seu processo ensino-aprendizagem ${ }^{11}$.

As percepções dos estudantes sobre seu ambiente de aprendizagem atual são um forte preditor de resultados de aprendizagem na universidade. Um ambiente que esteja de acordo com as preferências dos estudantes, oferece uma oportunidade para melhorar os resultados do processo ensinoaprendizagem. Alinhar o método de instrução com o ambiente de aprendizagem preferido dos estudantes ou diminuir a distância entre os ambientes de aprendizagem preferidos e percebidos pode ajudar os estudantes a melhorar mais o seu desempenho ${ }^{13}$.

Estudantes sugeriram maneiras de melhorar o ambiente de aprendizagem e o tratamento com eles durante o estágio, da seguinte forma: esclarecimento de papéis e expectativas; melhorar a comunicação; e apresentar os estudantes aos pacientes como membros da equipe para incentivar a aceitação dos mesmos pelos pacientes ${ }^{15}$.

\section{Categoria III - Fatores do ambiente educacional que influenciam no processo ensino- aprendizagem.}

Vários fatores do ambiente educacional podem influenciar no processo ensino-aprendizagem, sendo a estrutura física um deles, devendo haver espaço físico suficiente para que os móveis e equipamentos possam ser mudados de posição, se necessário; a disposição dos estudantes e docentes no recinto pode também exercer influência nos aspectos afetivos do ambiente educacional ${ }^{1}$.

A temperatura deve ser adequada, para que todos se sintam confortáveis, o que é especialmente digno de atenção quando há necessidade de se utilizar equipamentos de ar condicionado ou aquecedores. O ambiente físico deve também ser silencioso ou controlado, de acordo com as atividades propostas aos participantes; iluminação adequada ao tipo de atividade, permitindo que tudo seja visualizado sem maior esforço; recursos audiovisuais e tecnológicos de fácil acesso a todos, em bom estado e funcionando adequadamente ${ }^{1}$.

Outros estudos descrevem a insatisfação dos estudantes com a infraestrutura física das instituições que servem de campo de estágio curricular ${ }^{14}$. A falta de computadores dificultava aos estudantes o cumprimento das atividades propostas no ensino clinico. Devido a isso, os estudantes não tinham acesso às informações importantes dos pacientes que estavam registradas e descritas no prontuário eletrônico ${ }^{16}$

Em uma pesquisa, os estudantes relataram que não tinham certeza de suas funções no ambiente 
de ensino clínico, estavam preocupados com a carga de trabalho e com a orientação desejada para atender às demandas do estágio, resultando em situações variadas, desde gozo, maior confiança ou desenvolvimento do estudante ao desinteresse. Outra situação relatada foi a dificuldade em desenvolver relacionamentos com os membros da equipe.

Um ambiente educacional adequado deve proporcionar conforto ao estudante e prazer em participar das atividades educativas propostas, sentindo-se respeitado e seguro para expor as suas ideias, sem medo de ser criticado. Sentir-se pertencente ao grupo e fazendo parte importante dele, tendo a percepção clara que o seu trabalho e a sua participação contribuem para o aprendizado de todos. Proporcionando aos mais retraídos, encorajamento para expor as suas opiniões e pontos de vista, de modo que, ao longo do tempo, adquiram confiança para participar das atividades, em condições de igualdade com os demais ${ }^{1}$.

O docente é uma das peças mais importantes do ambiente educacional, sendo o responsável pela sua manutenção em condições favoráveis e pelo seu aperfeiçoamento, devendo ser vocacionados para a atividade didática e preparados para trabalhar com os estudantes ${ }^{1}$.

\section{CONCLUSÕES}

Ao término desta revisão integrativa, conclui-se que o ambiente educacional é o conjunto de elementos materiais e afetivos que circunda o estudante, sendo fatores ligados à instituição, ao docente e ao próprio estudantequedevem contribuir para a sua manutenção e aprimoramento. O ambiente deve proporcionar ao estudante sentir-se bem e confortável, com materiais e equipamentos adequados, estrutura adequada, docentes capacitados e motivados para ensinar.

Foi possível avaliar o ambiente/clima educacional de aspectos variados, e em diversos estudos, a percepção dos estudantes da área da saúde, suas insatisfações, satisfações, motivações, entre outros aspectos relacionados ao ambiente educacional em que estão inseridos.

E foi possível perceber o quanto o ambiente pode influenciar diretamente no processo ensinoaprendizagem do estudante, desestimulando, desmotivando-o em sua atividade diária.

A avaliação do ambiente/clima educacional é importante para fornecer dados e informações que podem ser utilizados para a tomada de decisões, visando o seu aperfeiçoamento e melhorando a qualidade do processo ensino-aprendizagem.

\section{REFERÊNCIAS}

1. Troncon LEA. Ambiente educacional. Med. 2014;47(3):264-71.

2. Serrano-gallardo P, Martínez-marcos M, Espejomatorrales F. Fatores associados à aprendizagem clínica dos estudantes de enfermagem nos cuidados de saúde primários: um estudo analítico transversal. 2016; 24(2803):1-13.

3. Messas JT, Leonello VM, Fernandes MFP, Gonçalves GCC, Bucchi SM, Mira VL. O ambiente educacional do curso de Graduação em Enfermagem na perspectiva dos estudantes. Rev da Esc Enferm da USP [Internet]. 2015;49(spe2):106-14. Disponível em: http://www.scielo.br/scielo.php?script=sci_ arttext\&pid=S0080-62342015000800106\&lng=pt\&tlng=pt

4. Hirsch CD, Barlem ELD, Barlem JGT, Silveira RS, Mendes DP. Fatores preditores e associados à satisfação dos estudantes de enfermagem. Acta Paul Enferm [Internet]. 2015;28(6):566-72. Disponível em: http:// www.scielo.br/scielo.php?script=sci_arttext\&pid=S010321002015000600566\&lng=pt\&tlng=pt

5. Stella RCR, Abdalla IG, Lampert JB, Perim GL, Aguilarda-Silva RH, Costa NMSC. Cenários de Prática e a Formação Médica na Assistência em Saúde. Rev Bras Educ Med. 2009;33(supl 1):63-9 .

6. Sem R. A percepção ambiental dos estudantes do ensino 
médio sobre o cuidado com a sala de aula. 2018;35(107):15667.

7. Mendes KDS, Silveira RCCP, Galvão CM. Revisão integrativa: método de pesquisa para a incorporação de evidências na saúde e na enfermagem. Texto Context Enferm. 2008;17(4):758-64.

8. Mendes KDS, Silveira RCCP, Galvão CM. Use of the bibliographic reference manager in the selection of primary studies in integrative reviews. Texto e Context Enferm. 2019;28:1-13.

9. Mogre V, Amalba A. Psychometric Properties of the Dundee Ready Educational Environment Measure in a Sample of Ghanaian Medical Students. 2019;16-24.

10. Hägg-Martinell A, Hult $\mathrm{H}$, Henriksson $\mathrm{P}$, Kiessling A. Medical students' opportunities to participate and learn from activities at an internal medicine ward: An ethnographic study. BMJ Open. 2017;7(2):e013046.

11. Orsini C, Evans P, Jerez O. Journal of Educational Evaluation for Health Professions How to encourage intrinsic motivation in the clinical teaching environment? a systematic review from the self-determination theory. 2015;10:1-10

12. Merighi MAB, Jesus MCP, Domingos SRF, Oliveira DM, Ito TN. Ensinar e aprender no campo clínico: perspectiva de docentes, enfermeiras e estudantes de enfermagem. Rev Bras Enferm. 2014;67(4):505-11.

13. Yeh $\mathrm{T}$, Huang $\mathrm{H}$, Chan WP, Chang C. Effects of congruence between preferred and perceived learning environments in nursing education in Taiwan : a crosssectional study. BMJ Open. 2016;6(5):1-8.

14. Mourão LF, Maria B, Teixeira V, Oliveira LB. Perspective of nursing students on curricular training. Revista de Enfermagem da UFPI. 2015;4(1):40-6.
15. Baecher-Lind LE, Chang K, Blanco MA. The learning environment in the obstetrics and gynecology clerkship: an exploratory study of students' perceptions before and after the clerkship. Med Educ Online. 2015;20(1):27273.

16. Hägg-Martinell A, Hult $\mathrm{H}$, Henriksson $\mathrm{P}$, Kiessling A. Medical students' opportunities to participate and learn from activities at an internal medicine ward: An ethnographic study. BMJ Open. 2017;7(2):1-11.

DATA DE SUBMISSÃO: 10/03/20 ～～DATA DE ACEITE: 01/05/20 